Célestinesca 32 (2008): 265-277 https://doi.org/10.7203/Celestinesca.32.20118

\title{
La Celestina en la Francia del Renacimiento y del Siglo de Oro: texto y contexto, difusión y fortuna
}

\author{
Florence Serrano \\ Christian-Albrecht - Universität Kiel
}

\begin{abstract}
A mediados del siglo xx, Gerard J. Brault, en la contraportada de su edición crítica de la primera edición francesa de La Celestina, lamentaba que el estudio de las relaciones literarias entre España y Francia fueran escasas $^{1}$. Hoy en día, a pesar de un interés reciente por las traducciones entre lenguas vernáculas, los mecanismos de difusión de los textos entre los diferentes países quedan por descubrir. Esta comunicación, basada en el estudio de la difusión de La Celestina en Francia, permitirá vislumbrar esta problemática sin perder de vista la necesidad de aportar «un nouvel éclairage» a los estudios celestinescos. Por tanto, nos hemos preguntado las causas y el porqué de que de La Celestina saliera una Célestine y las razones por las que los franceses crearon tres Célestine distintas en un siglo. Una vez analizadas la difusión y la recepción de La Celestina en Francia desde un punto de vista diacrónico, una breve comparación de las tres versiones en sus elementos más destacados permitirá percibir los contornos movedizos de La Célestine.
\end{abstract}

\section{Difusión y recepción de La Celestina en Francia: un análisis de la tradición impresa}

La historia de la obra en Francia se inicia mediante una alusión. En 1521 sale de las prensas francesas la traducción del Sylva nuptialis, un tra-

1.- "The chief foreign sources of the Renaissance in France were the literatures of antiquity and of Italy, and modern scholarship has naturally concentrated its efforts on the influence of classical and Italian authors upon the French literature of this period. Although it has long been recognized that Spain came next in importance in this respect, little attention has been devoted to Franco-Spanish literary relations during the Renaissance», Gerard J. Brault, Celestine. A critical Edition of the first French translation (1527), Detroit, Wayne State UP, 1963. 
tado misógino del italiano Giovanni Nevizanno, discípulo de Francisco Curtius y profesor de derecho en la universidad de Turin. En éste, Jean Nevizan, como lo llaman los franceses, cita una decena de veces la obra española. En sus anotaciones, apoya su argumentación antifeminista en las declaraciones de uno de los personajes de La Celestina: Sempronio. Su diatriba contra las mujeres en el primer Auto era conocida por los italianos, pero no por un francés que leyera dicho tratado en estos momentos. Es decir, una obra inspirada en otra podía parecer anterior. El azar de la difusión de los textos (aunque en este caso era lógico que antes se diera a conocer un texto latino que español) tiene como consecuencia un efecto de avance de La Celestina. No obstante, la obra de Nevizzano es un tratado jurídico y el hecho de que se imprima en Francia es quizá secundario, porque su difusión era realmente internacional en el terreno profesional del derecho, aunque no se puede negar que muchos jóvenes franceses estudiantes y menos jóvenes juristas conocieran La Celestina por este medio. Por una parte, Jean de Nevizan creó un "horizon d'attente» para el lector francés; por otra, la obra estaba relacionada con la Querelle des Femmes, la cual mantuvo un interés renovado en Francia. Jacqueline Hombrecher Ysquierdo, en su tesis sobre la imagen de La Celestina en la Francia del siglo XVI al siglo XX, estudia detenidamente el alcance de cada alusión de Nevizan y lamenta que el autor sólo evoque a Celestina "maestra de hazer virgos» y a Sempronio entre los «qui scribant mala de mulieribus» y concluye diciendo:

En somme, Nevizan se contentait de banaliser les thèmes motifs et sujets débattus par Fernando de Rojas, mais sans adhérer vraiment à un système de critique textuelle rigoureuse. C'est ainsi qu'il ne parvenait à nous transmettre que des aspects "flashs» de La Célestine, et n'offrira aux lecteurs du xviè siècle que des informations "clichés» par trop superficielle ${ }^{2}$.

Antes de ser difundida en Francia, La Celestina interesa más por los temas que contiene que por la novedad de su forma, lo que constituye un rasgo primordial para su difusión en el país galo.

Como acabamos de comentar, cuando en 1527 el lector francés, por no decir parisiense $e^{3}$, tiene acceso a La Célestine traducida por un desconocido, puede que no sea la primera vez que oye hablar de la obra. El inicio de la difusión textual de La Celestina en Francia puede compararse a la de

2.- Jacqueline Hombrecher Ysquierdo, 'La Celestina' en France (Xviè-Xxè siècles): Etude de réception critique et image, thèse de littérature comparée sous la direction de Daniel-Henri Pageaux, París III, 1989, p. 63.

3.- En las prensas de Galliot du Pré, que también se dedicará a editar traducciones de obras españolas como la Prison d'amour de Diego de San Pedro. 
Alemania ${ }^{4}$. En efecto, en los dos países se imprime una traducción basada en la versión italiana de Ordóñez ${ }^{5}$ durante los años veinte. Brault ha demostrado, en su edición crítica de esta traducción francesa ${ }^{6}$, que el traductor no sólo disponía de la versión italiana sino también de la española. El traductor justifica la traducción desde el italiano por el prestigio de dicha lengua, como expresa en el propio título: «Célestine en laquelle est traicte des deceptions des serviteurs envers leurs maistres \& des macquerelles envers les amoureux translate d'italien en francois» ${ }^{7}$. El traductor tenía la posibilidad de traducir directamente del original. ¿Por qué no fue así? Es Drysdall ${ }^{8}$ quien propone una respuesta a partir de la refutación de una tesis de Brault. Éste opina que el traductor desconocido es español por los numerosos hispanismos que utiliza:

There is a strong possibility that the translator's native language was Spanish and that these Hispanisms betray an incomplete command of French ${ }^{9}$.

Drysdall, en cambio, ve en estos hispanismos un rasgo del francés del primer tercio del siglo XVI, que conoce una fase de elaboración en parte gracias a neologismos inspirados en las lenguas vernáculas. Basándose en unos ejemplos, demuestra que el traductor tenía que ser francés:

However, in a period when borrowing was a widespread and deliberate, this is an unsafe criterion. On the other hand, it would seem that such mistakes as the following could only be made by a Frenchman:

$$
\begin{array}{ll}
\text { ¡Oyra el diablo! } & \text { (C-T 198 1. 10) } \\
\text { Il orra le diable. } & \text { (Brault 141, 1. 35 } 5^{10} \text { ) }
\end{array}
$$

Este ejemplo de Drysdall no es del todo satisfactorio. La traducción muestra más que una falta de conocimiento de la organización sintáctica de la frase castellana, una falta de contextualización de la frase de Pármeno. En efecto, éste muestra su descontento al ver a su amo dejarse engañar por Celestina. En cambio, no podemos negar que el segundo ejemplo de Drysdall refleje un conocimiento escaso del español

4.- Para un estudio de la recepción de La Celestina en Alemania, consultar la tesis doctoral de Fernando Carmona-Ruiz, http://ethesis.unifr.ch/theses/downloads.php?file=CarmonaF.pdf

5.- La primera edición sale a la luz a principios del 1506 en Roma, pero conoce mucho éxito, como lo revelan las multiples reediciones.

6.- Celestine. A critical Edition of the first French translation (1527), pp. 5-7.

7.- Celestine. A critical Edition of the first French translation (1527), p. 213.

8.- Denis L. Drysdall, La Celestine, in the French translation of 1578 by Jacques de Lavardin. A critical edition with introduction and notes, Londres, Tamesis, 1974.

9.- Celestine. A critical Edition of the first French translation (1527), p. 11.

10.- La Celestine, in the French translation of 1578, pp. 1-2, n. 2. 
Parmeno y Sempronio, en su cabo, departen. (C-T 203 11. 7-8)

Parmeno et Sempronio aussi pour autre part s'en vont. (Brault 145 1.5)

La traducción es claramente errónea y, además de basarse en una intuición, entra en contradicción con las frases siguientes: "Oyen gentes por la calle. Apercíbense para huir ${ }^{11} »$. Desde un punto de vista dramatúrgico, no pueden haberse ido si deciden escapar después. Todavía se puede añadir un argumento a favor de la tesis de Drysdall de que el traductor era francés. Durante los años de encarcelamiento de los miembros de la familia real francesa, era poco probable que un español colaborara con un editor francés. Es verdad que el acontecimiento de carácter político fue importantísimo para el fomento de las relaciones literarias, pero la influencia tardaría un poco más en hacerse notar en Francia ${ }^{12}$.

Se conocen cuatro ediciones de la primera traducción ${ }^{13}$. La primera ${ }^{14}$ tiene dos emisiones que presentan pocas diferencias (basicamente gráficas y de menor importancia ${ }^{15}$ ) y se omite "translate d'italien en francois» en la segunda. Se conservan tres ejemplares de cada emisión. El privilegio lleva la fecha del veintitrés de junio de $1527^{16}$. La segunda sale de las prensas el catorce de julio de 1529 en Lyon, es decir menos de un mes después de que el privilegio caduque. Parece ser que el editor Claude Nourry estaba ansioso por publicar la obra. En noviembre del mismo año, Jehan de Saint-Denis propone la tercera edición de esta primera traducción en París. La historia de esta primera versión francesa de La Celestina se acaba con su cuarta edición de 1542, que se compone nada menos que de cinco emisiones que corresponden a cada uno de sus editores: Nicolas Barbou, Jehan Fouchet, Madeleine Boursette (viuda de François Regnault II), Oudin Petit y Pierre Sergent. Ya son otros años, una vena hispanófila empieza a desarrollarse en la corte de Francisco I y el testimonio literario más claro estriba en algunas de las novelas cortas de Margarita de Navarra, ubicadas en España e influidas por la materia sentimental.

11.- Fernando de Rojas, La Celestina, ed. Dorothy S. Severin, Madrid, Cátedra, 2000, p. 255.

12.- Rojas se hace el portavoz de la importancia de relaciones políticas entre Francia y España al evocar la presencia de un embajador francés en el entorno de Celestina, como leemos en el auto I.

13.- Para las referencias de todas las ediciones francesas de La Célestine, consultar el Apéndice I de la edición crítica de Brault ya mencionada, pp. 213-218.

14.- Los datos conocidos no permiten establecer si se trata de la edición princeps.

15.- Es la opinión de Brault, quien no piensa en una posible segunda edición (si los cambios afectan la obra entera, por muy leves que sean). La segunda emisión sería entonces la primera reedición (falsificada si nos referimos al privilegio) y por consiguiente tendríamos cinco ediciones de la primera traducción. Le agradezco a José Luis Canet el haberme comentado esta hipótesis.

16.- Celestine. A critical Edition of the first French translation (1527), p. 17. 
En cuanto a la segunda traducción, quizás la más famosa de las tres, es la única de la que conocemos a su traductor, Jacques de Lavardin. Drysdall resuelve finalmente las posibles dudas sobre su personalidad ${ }^{17}$. Se basa en la genealogía establecida por Vauloger en 1888, que encaja con los testimonios que tenemos sobre su biografía, para demostrar que al "seigneur du Plessis Bourrot, du Plessis Auzouer et de la Haye de Roullée, escuyer et échanson du roi de Navarre Antoine de Bourbon» le fue encargada una traducción de La Celestina por su padre Louis, quien muere hacia 1555. En esas fechas, Drysdall estima que Jacques tiene como mucho treinta años. Parece ser que no cumplirá con la voluntad de su padre hasta unos veinte años después, al volver de un primer viaje a Roma donde disfrutó de la compañía de "[s]on amy» du Bellay entre 1553 y 1557. Sabemos también que tuvo una hija llamada Suzanne, detalle que, más allá de lo anecdótico, justifica tal vez el propósito didáctico que declara en su traducción. Por último, fue uno de los nobles que participaron en la guerra civil de 1578 y no tuvo más remedio que exiliarse en sus tierras. Pero gracias a ese "si long et ennuyeux exil» tendrá la oportunidad de dedicarse a las traducciones. En la segunda edición de su Célestine, se incluye una carta firmada por él, fechada el uno de julio de 1597, último testimonio que conservamos suyo.

De los trabajos de Lavardin, han llegado hasta nosotros dos traducciones además de La Célestine: una del latín de Marin Barlece, titulada Histoire de Georges Castriot, surnommé Scanderberg, que tuvo mucho éxito en su época (a partir de 1576, fecha de la primera edición) y otra del italiano de Flaminio de Nobili, llamado le Traitté de l'amour humain ${ }^{18}$ de 1588 . Es probable que, como su colaborador Florent Chrestien, autor de una elegía incluida en su Célestine, se haya entretenido escribiendo versos, ya que convivía con los miembros más destacados de la Pléiade, como el ya mencionado Du Bellay o Ronsard, quien compone un soneto que se imprime en los preliminares de la segunda edición de l'Histoire de Georges Castriot. En resumidas cuentas, si tuvo que llevar una vida retirada de erudito, por no decir bibliófilo — que recuerda la de Montaigne-, fue a su pesar, ya que se acomodó en un entorno cortesano.

Detengámonos en la fecha de 1578, año de la primera edición de la versión de Lavardin, según defiende con prudencia Drysdall en su edición crítica, a diferencia de Penney y Brault ${ }^{19}$ :

It is very unlikely that there was ever an edicion earlier than 1578, since the only evidence for those of 1560 ,

\section{7.- La Celestine, in the French translation of 1578, p. 9.}

18.- La traducción de la misma obra titulada Traicté de la Nature d'amour que se imprime en 1579 sería de otro traductor, según Drysdall, y el privilegio atribuido a la traducción de Jacques de Lavardin es de 1587.

19.- Respectivamente, p. 117 y p. 215. 
1567 and 1577 comes from book-sellers'catalogues. The date of the privilege moreover seems conclusive: «le xix. iour de Mars, l'an de grace, mil cinq cens soixante \& dixhuict» ${ }^{20}$.

Afirma a continuación que no existen más de tres ediciones de esta traducción de Lavardin:

The three authenticated editions are those of Paris, for Gilles Robinot, 1578, and by Nicolas Bonfons, without date, and of Rouen, by Théodore Reinsart and Claude Le Villain, 1598, re-issued by the latter in 1599.

La primera de ellas contiene un privilegio fechado el 21 de mayo, que protege los «derechos del editor» durante seis años «iusques au terme de six ans». Así llegaríamos al año 1584, en el que suponemos que se imprimió la edición de Bonfons. La hipótesis de que pudiera ser anterior y, por tanto, ilegal justificaría la ausencia de fecha. No obstante, el hecho de que las dos ediciones sean parisinas supone un riesgo importante, ya que el control del mercado librero por el poseedor del privilegio le sería más fácil. Además, un editor como Bonfons, que imprimió entre otras obras una versión francesa de Grisel y Mirabella de Juan de Flores, no tenía razones para arriesgarse (a no ser que comprara al antiguo editor su derecho de impresión). Así pues, no tenemos ningún otro dato relevante para determinar la fecha ad quem. Esta edición incluye "La Vieille Courtizane» de du Bellay, pero omite el poema en latín de Morillon. Las tres reimpresiones posteriores ruanesas, basadas en la edición de Nicolas Bonfons, son idénticas (menos en lo que atañe al título) y presentan cierto número de incorrecciones.

Acabada la historia de la difusión de La Celestina en el siglo XVI, llegamos finalmente a la tercera y última versión premoderna de la obra. He indicado que la última edición de la versión de Lavardin fue impresa en Ruán en los postrímeros años del siglo dieciséis. En 1633, aparece en dicha ciudad, conocida por sus intercambios con España ${ }^{21}$ (ora comerciales, ora literarios), una versión bilingüe de La Celestina salida de las prensas de Charles Osmont. Presenta el texto español y «en miroir» el texto francés. El mismo año, ve la luz una segunda edición idéntica e impresa por Carlos Labayen en Pamplona. Este editor ya había publicado una versión de La Celestina (en español) en Zaragoza en 1607 con la colaboración de Juan de Larumbe ${ }^{22}$. El año siguiente se vuelve a imprimir en Ruán. En 1644, la

20.- La Celestine, in the French translation of 1578, p. 24.

21.- Consultar, por ejemplo, el artículo de Natalia Muchnik, "Du catholicisme des judéoconvers: Rouen, 1633», Revue XVII Siècle, 58 (2006), pp. 277-299.

22.- Clara Louisa Penney, The Book Called 'Celestina' in the Library of the Hispanic Society of America, Hispanic Society of America, New York, 1954, pp. 74-76. 
última edición de La Célestine y de La Celestina a la vez aparece de nuevo en Ruán. No volverá a imprimirse la versión francesa ni tampoco la original hasta el siglo XIX. Es de recalcar que esta traducción de La Célestine ha podido favorecer la difusión de La Celestina en su país de origen durante el Siglo de $\mathrm{Oro}^{23}$. Sin embargo, puede que ese ímpetu franco-español haya fracasado. Resulta relevante la opinión de Menéndez y Pelayo, justificando el fin de la difusión de La Celestina en España, fundándose en términos estéticos:

[...] la principal razón hubo de ser el cambio del gusto, la exuberancia de la producción dramática y novelesca, que había llevado al ingenio español por otros rumbos y ofrecía a los hombres del siglo xvil alimento más adecuado a sus inclinaciones. La Celestina era todavía compatible con el arte de Cervantes, de Quevedo, de Lope, de Tirso, puesto que le contenía en germen, pero no era compatible con los Góngoras, Calderones y Gracianes. Cuando triunfaron los cultos, los discretos y sutiles, y se prefirió el estilo almidonado a la ejecución franca y vigorosa, pocos paladares pudieron gustar con deleite aquel fruto sabrosamente agrio del árbol nacional ${ }^{24}$.

Menéndez y Pelayo añade, a modo de colofón, que la obra fue formalmente expurgada conforme al «Index Expurgatorius» de 1632 de la Inquisición. En fin, si la difusión en Francia se vale del éxito inicial de $\mathrm{La}$ Celestina ${ }^{25}$, su éxito en Francia es también un factor positivo de difusión en España, independientemente de las circunstancias.

\section{Un análisis comparativo del paratexto de las tres versiones francesas de La Celestina: unas breves anotaciones}

Intentaré analizar en este apartado las diferencias existentes en el paratexto para poder determinar, aunque de forma rápida, las estrategias de los traductores y/o de los editores, las cuales pueden condicionar su

23.- Ediciones españolas: la de Zaragoza, 1607, y tres de Madrid, en 1601, 1619 y 1632.

24.- La Celestina: Razones para tratar de esta obra dramática en la historia de la novela española, Marcelino Menéndez y Pelayo, Biblioteca Virtual Miguel de Cervantes, 1999, razón 14, http://descargas.cervantesvirtual.com/servlet/SirveObras/p194/02588385479192984197857/ 029164_0003.pdf.

25.- Sin embargo, el éxito no constituye un factor determinante para la difusión en el extranjero. Podemos dar el ejemplo de Grimalte y Gradissa de Juan de Flores, la continuación de la Elegia di Madonna Fiammetta de Boccaccio, que fue más leída en Francia que en España si nos basamos en el número de impresos (a pesar de que el argumento no sea del todo satisfactorio si pensamos que un gran número de ejemplares conservados señala muchos invendidos y con lo cual un éxito menor). 
recepción. Es decir, por una parte, imaginando que tenemos entre manos los impresos descritos, podríamos preguntarnos qué elementos destacarían a primera vista al posible lector; por otra parte, qué diferencia hay entre la tradición española y francesa para la edición de La Celestina. Los aspectos elegidos en este trabajo son los títulos y las reflexiones sobre el género reivindicado por los editores, así como el nombre de los personajes.

A diferencia de la versión de Lavardin y de la tercera traducción, la primera presenta un título que carece del artículo definido: Célestine en laquelle est traicte des deceptions des serviteurs envers leurs maistres \& macquerelles envers les amoureux translate d'italien en francois. Más allá de un problema de introducción del título en el discurso, aspecto que permanece hasta en la actualidad, como demuestran las fluctuaciones en nuestras citas, podemos pensar que el grado de particularización es inferior en ausencia de determinación, es decir que el locutor no espera del destinatario un conocimiento previo de la entidad constituida por "Célestine». La adición del artículo por parte de Lavardin podría remitir de manera más que implícita a su voluntad de erigir la obra a nivel de referencia cultural común. Sin embargo, un nombre propio no sigue las normas de la determinación ya que no las necesita. Los títulos de las versiones españolas e italianas anteriores son Tragicomedia de Calixto y Melibea o Libro de Calixto y Melibea y dela puta vieja Celestina. No obstante, en 1569 en Alcalá, se preparan dos ediciones cuyo título es [Celestina] tragicomedia de Calisto y Melibea. En 1571, en Cuenca, otra edición tiene el mismo título, esta vez sin corchetes. En España también se conocía «vulgarmente» el libro como Celestina en aquella época. En las ediciones de Ruán y de Navarra de 1633, al cambio de apelativo le corresponde un comentario en la portada en castellano: Tragicomedia de Calisto y Melibea, vulgarmente llamada Celestina en la qual se contienen (de mas de su agradable y dulce estilo) muchas sentencias filosofales, y avissos muy necessarios para mancebos, mostrandoles los engaños que están encerrados en sirvientes, y alcahuetas. En España, el título que se emplea más es el que se basa en el género al que se refiere, es decir la tragicomedia. El comentario indica que se acepta el título de Celestina pero que su uso no es aconsejable ya que remite al vulgo. Pero no hay que perder de vista que el adverbio «vulgarmente» no tiene un sentido despectivo y que significa "comúnmente». Sin embargo, el título francés de la misma edición es La Célestine ou histoire tragicomique de Caliste et de Melibee. Parece ser que el título corresponde a una estrategia comercial, busca un público determinado, por lo que la edición se sitúa en el devenir de las ediciones antecedentes. El nombre correspondiente al género se transforma en adjetivo, es decir un registro literario. Sugiere que en Francia el género no puede valer de argumento de venta para la obra. En efecto, en Francia el género no consigue atraer a un público (porque el teatro francés no ha conocido una renovación dramática como la española y se 
inspira más en la Commedia dell'arte que en el teatro hispano del Siglo de Oro) y la única obra maestra perteneciente a este género es tal vez Le Cid de Corneille, obra creada en 1637. Una obra aislada no será suficiente para garantizar un interés por la forma tragicómica a largo plazo. Pero reconozcamos que ese término tampoco se refiere a un género perenne en España. En cambio, el género de "1'histoire» ${ }^{26}$ conoce un gran éxito en esta misma época y 1633 es precisamente el año en el que se revisa por segunda vez L'Histoire comique de Francion de Charles Sorel. La ventaja de la tercera traducción de La Celestina es que se adapta a la petición de un doble público. Por un lado, la traducción es elegante y muy cercana al texto español (ya que servía de manual didáctico de la lengua española para los francohablantes y viceversa). Por otro, muestra la influencia entre los dos países desde un punto de vista editorial, mediante la mención en la portada de su autoría en la edición ruanesa, al igual que en la edición madrileña de 1631, a cargo de la viuda de Alonso Martín: «por el bachiller Fernando de Rojas $»^{27}$. Brault recalca el valor de esta traducción a pesar de que sea la última de las tres:

The third French version produced during this period is probably the best translation of the Celestina. In general, experts have neglected to consult it when attempting to resolve problems of interpretation, preferring to study the Ordóñez or Lavardin renderings ${ }^{28}$.

Tradicionalmente, la crítica ha preferido interesarse en las primeras ediciones, prefiriendo la antigüedad a la calidad de la versión. Nuestras futuras investigaciones se centrarán en este aspecto. Sin embargo, los críticos se apoyaron varias veces en el título de la traducción de Lavardin para destacar su dimensión didáctica, vinculada al tópico de la reprobatio amoris:

La Célestine fidellement repurgee, et mise en meilleure forme par Jacques de Lavardin, Escuyer, Seigneur du Plessis Bourrot en Touraine. Tragicomedie jadis Espagnole, composee en reprehension des fols amoureux, lesquels vaincuz de leurs desordonnez appetis invoquent leurs amies, \& en font un Dieu: aussi pour descouvrir les tromperies des macquerelles, e l'infidelite des meschans \& traistres serviteurs.

26.- También se puede mencionar L'Histoire aethiopique de Heliodorus, contenant dix livres, traictant des loyales et pudiques amours de Théagènes Thessalien et Chariclea Aethiopienne traducida por el humanista Jacques Amyot e impresa por primera vez en 1547. Pero la influencia de las novelas griegas todavía se hace sentir en la producción novelesca de "l'Âge classique» francés, por ejemplo en las novelas de Mme de La Fayette.

27.- Clara Louisa Penney, The Book Called 'Celestina', pp. 79-83.

28.- Celestine. A critical Edition of the first French translation (1527), p. 4. 
Más allá del empleo del determinante anteriormente analizado, se nota un intento de apropiación, por no decir de aculturación de la obra española. En efecto, aquí la traducción supone un cambio de idioma y de identidad nacional, con lo cual esta visión se opone al concepto moderno de traducción. El primer traductor también intentó transformar la obra española en obra francesa, quitando las indicaciones escénicas así como el empleo novedoso de los apartes mediante el uso de los paréntesis y omitiendo las indicaciones espaciales, elaborando así una posible intriga sucedida en Francia. Los traductores franceses se sentían incómodos por el hibridismo genérico. Por ejemplo, Lavardin decide suprimir los argumentos de cada auto.

La distribución de los nombres de los personajes es otro testimonio de la libertad que se tomó Jacques de Lavardin. Pero, al menos, tenemos que reconocer la sinceridad que manifiesta acerca de su lector al justificar sus cambios:

Amy lecteur, recontrant quelques noms des entreparleurs de ceste Tragicomedie changez, comme trop ressentans leur Comique Latin, ie te supplie ne t'en offenser ${ }^{29}$.

Lavardin pretende borrar la intertextualidad posible con la comedia de Terencio y Plauto, lo que justificaría aparentemente los cambios de "Malican» por Sempronio, "Corneille» por Pármeno, «Bezane» por Sosia y «Carabin» por Traso. Además, Drysdall expresa su perplejidad al haber leído «Boniface» en vez de Pármeno, cuando Elicia evoca su muerte en el decimoquinto auto. En la primera traducción, los nombres no cambiaban radicalmente, pero algunos fueron traducidos como Célestine, Melibee y Lucresse (o Lucrece). En la última versión, el nombre de Calisto también se traduce, pero únicamente en el título aparecerá Caliste. Las fluctua iones gráficas en una misma obra también conciernen a los nombres. He entresacado el ejemplo de Mirabella, que el traductor italiano del Grisel y Mirabella bautiza Isabela y que se transforma en Isabel en algunas de las traducciones francesas. Drysdall remite al estudio de Cejador y Frau$\mathrm{ca}^{30}$ sobre los orígenes de estos nombres, donde demuestra que dichos cambios recuerdan la comedia plautina y terenciana. Las variaciones en los nombres tienen un alcance limitado y se vinculan normalmente a una tradición nacional o literaria.

29.- La Celestine, in the French translation of 1578, p. 44.

30.- La Celestine, in the French translation of 1578, p. 23. 


\section{Conclusión}

A raíz de lo expuesto, creo que la historia de la difusión de La Celestina en Francia es innegablemente compleja y siguió varias fases. La primera traducción sirve de introducción del texto en Francia. La segunda pretende adaptar la obra para recalcar su didactismo (si consideramos que existe en La Celestina). La tercera presenta una traducción elegante en una edición bilingüe. Esta última versión se integra en la producción francesa de la época y fomenta el aprendizaje de los idiomas. Otras obras conocen una difusión parecida, caso de Grisel y Mirabella de Juan de Flores, con la misma interpolación italiana entre la versión original y la francesa, las cuales también fueron utilizadas como manuales de aprendizaje lingüístico.

Un análisis textual preciso entre las tres traducciones queda por realizar, al igual que un estudio sobre las influencias de La Celestina en la producción francesa, tanto desde un punto de vista temático como genérico. El criterio de éxito no justifica la elaboración de una traducción al francés; habría, pues, que determinar aquello que en la producción francesa contemporánea justificó el interés por La Celestina. Las novelas sentimentales y La Celestina tienen en común presentar lo que se llamará en Francia «les histoires piteuses». Tal vez ésa sea una de las claves de su exportación hacia Italia y Francia. 


\section{Bibliografía consultada}

Brault, Gerard J., Célestine. A critical Edition of the first French translation (1527), Detroit, Wayne State UP, 1963.

Carmona-Ruiz, Fernando, La recepción de "La Celestina» en Alemania en el siglo XVI, tesis doctoral dirigida por Hugo O. Bizzarri, Friburgo (Suiza), 2007.

Drysdall, Denis L., La Célestine, in the French translation of 1578 by Jacques de Lavardin. A critical edition with introduction and notes, Londres, Tamesis, 1974.

Hombrecher Ysauierdo, Jacqueline, 'La Celestina' en France (XVì̀-XXè siècles): Etude de réception critique et image, tesis doctoral dirigida por DanielHenri Pageaux, París III, 1989.

Menéndez y Pelayo, Marcelino, La Celestina: Razones para tratar de esta obra dramática en la historia de la novela española, Biblioteca Virtual Miguel de Cervantes, 1999, razón 14, http://descargas.cervantesvirtual.com/ servlet/SirveObras/p194/02588385479192984197857/029164_0003.pdf.

Penney, Clara Louisa, The Book Called 'Celestina' in the Library of the Hispanic Society of America, New York, Hispanic Society of America, 1954.

RojAs, Fernando de, La Celestina, edición de Dorothy S. SEverin, Madrid, Cátedra, 2000.

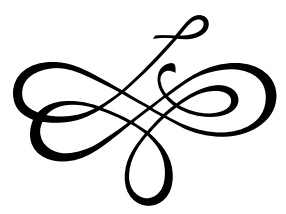




$$
\text { I }
$$




\section{Serrano, Florence, "La Celestina en la Francia del Renacimiento y del Siglo de Oro: texto y contexto, difusión y fortuna", Célestinesca, 32 (2008), pp. 265-277.}

\section{RESUMEN}

La llegada de La Celestina a Francia ocurre en 1527 con una primera traducción anónima. Las múltiples ediciones posteriores revelan el éxito que conoce la obra adaptada al gusto del país destinatario. Medio siglo después, Jacques de Lavardin propone una segunda traducción, o bien adaptación libre por no decir glosa, que se inspira todavía más en la versión italiana. El propósito del traductor es claramente didáctico y el texto no deja de ser leído hasta los postreros años del siglo. Una última traducción anónima sale a la luz en 1633 en una edición bilingüe. El estudio diacrónico de la difusión de la Tragicomedia más allá de los Pirineos nos permite reflexionar sobre lo que condiciona el interés de los franceses. No obstante, un análisis comparativo de las versiones determina los rasgos de "Las Celestinas" que los franceses llegaron a conocer a lo largo de los siglos XVI y XVII. Se plantean, finalmente, las consecuencias que tuvieron estas ediciones en la producción francesa que les fue contemporánea.

Palabras ClaVe: Difusión, Francia, Renacimiento, Siglo de Oro, traducción.

\section{ABSTRACT}

French Renaissance readers were able to read La Célestine which was translated from Spanish from 1527 on. The multiple editions of this literary work by Fernando de Rojas testify to the success that it knew in the $16^{\text {th }}$ century in Valois France. Contemporaries of Montaigne can read Jacques de Lavardin's version, a text which was adapted, rather than translated from the original Spanish. Inconsistencies with the Italian version are numerous and work to illustrate the diffusion throughout Europe of many Castilian works. A few years before the production of Le Cid, in 1633, the last translation of La Célestine is printed in Rouen and in Pamplona in a bilingual edition which is presented with the two languages side by side. A study carried out from a diachronic point of view and a comparative analysis of different French versions allow us to measure the success of La Celestina in France and to understand the interest that it stirs up. Moreover, La Celestina outside of Spain appears as polymorphous work that has known many «births».

KEY WORDS: Diffusion, France, $16^{\text {th }}$ century, $17^{\text {th }}$ century, translation. 